\title{
Identification of metabolism genes related to hepatocarcinogenesis and progression in type 2 diabetes mellitus via co-expression networks analysis
}

\author{
Yiming Bi ${ }^{1} \mathbb{0}$, Bei Yin ${ }^{1}$ and Guanjie Fan² ${ }^{*}$
}

\begin{abstract}
Background: Type 2 Diabetes Mellitus (T2DM) is an independent risk factor of hepatocellular carcinoma (HCC). However, the related genes and modules to hepatocarcinogenesis and progression in T2DM remain unclear.

Methods: The microarray data from Gene Expression Omnibus (GEO) were analyzed to screen differentially expressed genes (DEGs) of T2DM and HCC dataset. Then, weighted gene co-expression network analysis (WGCNA) was performed on these DEGs to detect the modules and genes, respectively. Common genes in modules with clinical interests of T2DM and HCC were obtained and annotated via GOSemSim package and Metascape. Genes related to late-stage HCC and high glycated haemoglobin ( $\mathrm{HbA} 1 \mathrm{c}$ ) were also identified. These genes were validated by UALCAN analysis and univariate cox regression based on The Cancer Genome Atlas (TCGA). Finally, another two independent datasets were applied to confirm the results of our study.

Results: A total of 1288 and 1559 DEGs of T2DM and HCC were screened, respectively. Kyoto Encyclopedia of Genes and Genomes (KEGG) enrichment revealed several shared pathways in two diseases, such as pathways in cancer and metabolism. A total of 37 common genes correlated with T2DM and HCC were then identified with WGCNA. Furthermore, 12 genes from modules associated with late-stage $\mathrm{HCC}$ and high $\mathrm{HbA1c}$ were regarded as hub genes. Among these genes, 8 genes associated with tumor invasion and metastasis were validated by UALCAN analysis. Moreover, downregulations of ACAT1, SLC2A2, PCK1 and ABAT were significantly associated with poorer prognosis in HCC patients with elevated HbA1c. Additionally, the expressions of $P C K 1$ and $A B A T$ were raised in HepG2 cells pre-treated with metformin and phenformin.
\end{abstract}

Conclusions: The present study confirmed several metabolic genes related to hyperglycemia and malignant tumor, which may provide not only new insights into the pathogenesis of hepatocarcinogenesis and progression in T2DM, but also novel therapeutic targets for T2DM patients with HCC in the future.

Keywords: Type 2 diabetes mellitus, Hepatocellular carcinoma, Metabolism genes, WGCNA

\footnotetext{
*Correspondence: fanfanfangj@163.com

2 Department of Endocrinology, Guangdong Provincial Hospital

of Chinese Medicine, the Second Affiliated Hospital of Guangzhou

University of Chinese Medicine, Guangzhou, China

Full list of author information is available at the end of the article
}

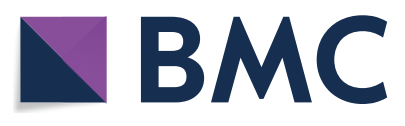

(c) The Author(s) 2021. This article is licensed under a Creative Commons Attribution 4.0 International License, which permits use, sharing, adaptation, distribution and reproduction in any medium or format, as long as you give appropriate credit to the original author(s) and the source, provide a link to the Creative Commons licence, and indicate if changes were made. The images or other third party material in this article are included in the article's Creative Commons licence, unless indicated otherwise in a credit line to the material. If material is not included in the article's Creative Commons licence and your intended use is not permitted by statutory regulation or exceeds the permitted use, you will need to obtain permission directly from the copyright holder. To view a copy of this licence, visit http://creativeco mmons.org/licenses/by/4.0/. The Creative Commons Public Domain Dedication waiver (http://creativecommons.org/publicdomain/ zero/1.0/) applies to the data made available in this article, unless otherwise stated in a credit line to the data. 


\section{Background}

Type 2 diabetes mellitus (T2DM) is one of the most common chronic diseases worldwide. According to International Diabetes Federation (IDF), diabetes affected an estimated 463 million people worldwide in 2019, accounting for 9.3 percent of adults aged 20 to 79 , with a predicted increase to 10.9 percent by 2045 [1]. Besides eliciting metabolic disorders and vascular damages, T2DM is a major predisposing factor of cancers, predominantly in gastrointestinal malignancies, including hepatocellular carcinoma (HCC) [2]. Accordingly, approximately 7 percent of new cases of $\mathrm{HCC}$ can be attributed to diabetes, and the incidence of HCC in patents with T2DM is about twofold higher than that in normal individuals $[3,4]$. The risk of HCC development is also positively correlated with T2DM duration, which significantly increases to 7.52 times in individuals with a 10 -year-duration of diabetes [5].

Furthermore, there is also strong evidence indicating the relationships between the rise of the incidence of T2DM and the risk of deaths from HCC [6]. A metaanalysis showed that the presence of diabetes decreased overall survival rate among patients with $\mathrm{HCC}$; and the mortality for $\mathrm{HCC}$ was 2.5 -fold higher in T2DM patients [7]. A number of studies also reported that treatment with glucose-lowering medications like metformin could improve the survival rate of patients with $\mathrm{HCC}$ [8]. These shreds of evidence revealed that T2DM had turned into an independent risk factor for HCC development.

The underlying mechanisms involved in T2DM and HCC are complicated, including hyperglycemia, insulin resistance, and inflammatory [9]. Endogenous insulin acting on liver activates a number of signaling pathways such as insulin-like growth factor (IGF) signaling pathway, phosphatidylinositol 3 kinase (PI3K) pathways and mitogen-activated protein kinase (MAPK) pathways that contribute to hepatic cell proliferation and tumor progression [10]. Further, persistently high blood glucose and circulating insulin levels accelerate the secretion of inflammatory factors and the accumulation of metabolites such as free fatty acids (FFA) and glycation end products (AGEs). As a result, hepatic stellate cells are activated and liver fibrosis is promoted [11]. Chronic hyperglycemia also increases the frequency of $K R A S$ and $M Y C$ variants, possibly because of nucleotide imbalance [12]. Although the metabolism disorders of T2DM have been reported to play crucial roles in stimulating liver cancer growth, the specific pathological mechanisms and key genes of these processes remain vague.

Weighted gene co-expression network analysis (WGCNA) is a novel biological method widely utilized in the high-throughput sequencing data analysis. It focuses on the intramodular connectivity and gene significance, which alleviates the multiple testing problem inherent in microarray [13]. Based on the co-expression networks, the hub genes for relating modules to one another and to external clinic traits are identified. This method has been successfully applied to identify shared pathogenesis of 2 diseases. For instance, Zhu et al. used WGCNA to reveal ten hub genes involved in the development of Alzheimer's disease and T2DM [14]. Bi et al. also employed WGCNA to demonstrate a novel biomarker for distinguishing alcohol-associated HCC from non-alcohol-associated HCC [15]. Thus, to fully understand T2DM and HCC, we utilized this method to search for genes acting on metabolism disorders in T2DM and development in HCC.

\section{Materials and methods \\ Data preparation}

Gene expression datasets GSE38642, GSE44035 and GSE25724 were downloaded from GEO, which contains 70 normal samples and 16 T2DM samples. Meanwhile, GSE101685 was collected, including 8 normal samples and $24 \mathrm{HCC}$ samples. All data in different samples were normalized by quantity prior to performing gene differential analysis with limma $R$ package [16]. Differentially expressed genes (DEGs) with $\mathrm{p}$-value $<0.05$ were screened. DEGs with $\mid \log$ fold change $(\operatorname{logFC}) \mid>0.2$ in $\mathrm{T} 2 \mathrm{DM}$ and $|\operatorname{logFC}|>1$ in $\mathrm{HCC}$ were further investigated in our study. In addition, two independent datasets, GSE50397 and GSE69850, were downloaded to validate the results of the present study. GSE50397 provided 89 samples with different levels of blood glucose (HbA1c), while GSE69850 provided an evaluation of changes in gene expression associated with the treatment of human HepG2 cells with 34 different chemical compounds, including metformin and phenformin.

\section{Gene ontology (GO) and KEGG analysis of DEGs}

In order to explain the biological functions and further interactions, both DEGs of T2DM and HCC were annotated by GO analysis and KEGG pathways analysis. The $p$ value $<0.05$ was set to be statistically significant, with the top 10 visualized in $R$.

\section{Weighted gene co-expression networks (WGCNA) and module analysis}

The DEGs of T2DM and HCC utilized WGCNA R package to construct co-expression networks with corresponding clinic traits, respectively [13]. Data were checked to identify the outliers in the samples. All samples from T2DM dataset were well clustered, while one offending sample was removed in HCC dataset. The soft-thresholding power identified by pickSoftThreshold function was applied to the automatic network construction. The result was clustered by topological overlap 
matrix analysis, containing module assignments labeled in colors and module eigengenes (MEs). In addition, the correlations between MEs and clinic traits were calculated via Pearson's correlation test. The modules with a $|\mathrm{ME}|>0.3$ and a $p$-value $<0.05$ were considered momentous in the interactions with clinical features [17].

\section{Correlation analysis between clinically significant modules in T2DM and HCC}

Furthermore, genes relevant to T2DM and HCC within each module were annotated via GO biological process (BP) enrichment. Different sets of GO terms in modules of T2DM and HCC were collected. Since the functions of genes can be enriched by GO terms, their biological function similarities can also be assessed by aggregating similarities of GO term sets. Therefore, the biological correlations between T2DM and HCC modules were determined by the calculation of $\mathrm{GO}$ semantic similarities via DOSE R package [18]. The result with a correlation score $\geq 0.5$ was defined as significant, indicating that there was a certain correlation in biological function between the two modules.

\section{Common genes identification and analysis in T2DM and HCC}

The DEGs within related modules were analyzed with Venn tool (https://www.bioinformatics.org/. psb.ugent. be /webtools/Venn/) to define the common upregulated and downregulated genes in T2DM and HCC. Common genes involved in the pathogenesis of T2DM and HCC were interpreted by Metascape (last updated on 2021-0201) [19], a website tool integrating ontology sources such as GO biological process, KEGG pathways, Reatome gene sets, Canonical Pathways, DisGeNET and PaGenBase.

\section{Prognostic genes identification and validation}

In addition, the correlations between cancer progression and HbA1c abnormality were also explored. Genes in corresponding modules were screened from common genes in T2DM and HCC. With a gene significance (GS) $>0.2$ and module membership (MM) $>0.8$ [17], hub genes were selected and further validated by UALCAN analysis based on TCGA database (http://ualcan.path.uab.edu/analysis. html) [20]. Univariate Cox regression was also applied to detect high risk factors in HCC. Moreover, the protein samples of prognostic genes were also validated via the Human Protein Atlas (http://www.proteinatlas.org).

\section{Hub genes confirmation via external independent data}

To confirm the correlations with prognostic genes and glucose control, genes were analyzed in an independent dataset GSE50397. According to the HbA1c value, T2DM samples were divided into 2 subunits (HbA1c $>6.5 \%$ and $\mathrm{HbA} 1 \mathrm{c} \leq 6.5 \%$ ), and one-way ANOVA was made to compare the genes expressions among normal, and the 2 sub-units of T2DM. Finally, the correlations between hyperglycemia and prognostic genes were also validated through HepG2 samples prepared with hypoglycemic agents.

\section{Results}

\section{Identification and functional enrichment of DEGs}

A total of 1288 DEGs were screened between T2DM and normal controls, whereas 1559 DEGs were obtained between HCC and the normal samples. Of these DEGs, 440 genes were upregulated and 848 were downregulated in T2DM, while 618 were upregulated and 941 were downregulated in HCC (Fig. 1a and b). As indicated by GO analysis, DEGs in T2DM were mainly enriched in reproductive structure development and cell junction organization (Fig. 1c). On the other hand, metabolic processes involving oranic acid, carboxylic acid and small molecules were observed in HCC (Fig. 1d). KEGG analysis exhibited several shared pathways concerning T2DM and HCC. The upregulated DEGs in T2DM and HCC were enriched in pathways in cancer and extracellular matrix (ECM)-recptor interaction, whereas the downregulated DEGs were enriched in metabolic pathways and amino acids degradation (Fig. 1e and $\mathrm{f}$ ). In brief, both GO and KEGG analyses strongly demonstrated the correlations between T2DM and HCC, since the expression of genes involved in cellular metabolic was significantly reduced, and the expression of genes associated with cell development was substantially increased. The detailed information of functional enrichment of DEGs is shown in Supplementary Table S1 to S6.

\section{Co-expression networks construction and modules correlation between T2DM and HCC}

WGCNA was utilized to screen gene clusters with similar biological functions and construct the correlations between gene clusters and specific clinic traits. A soft power of $\beta=7$ was set and eventually a total of 9 gene clusters, also known as modules, were identified in T2DM. Additionally, a total of 7 modules in HCC were obtained with a soft power of $\beta=8$. The gray module represented the non-clustering genes and was excluded. Genes in yellow, blue, turquoise, brown and green modules were negatively correlated with T2DM, while those in black, pink and red modules were positively correlated (Fig. 2a and b). The result of HCC demonstrated that all modules except for the turquoise one were negatively correlated with HCC (Fig. 2c and d). The top $10 \mathrm{GO}$ BP terms of each module in 2 

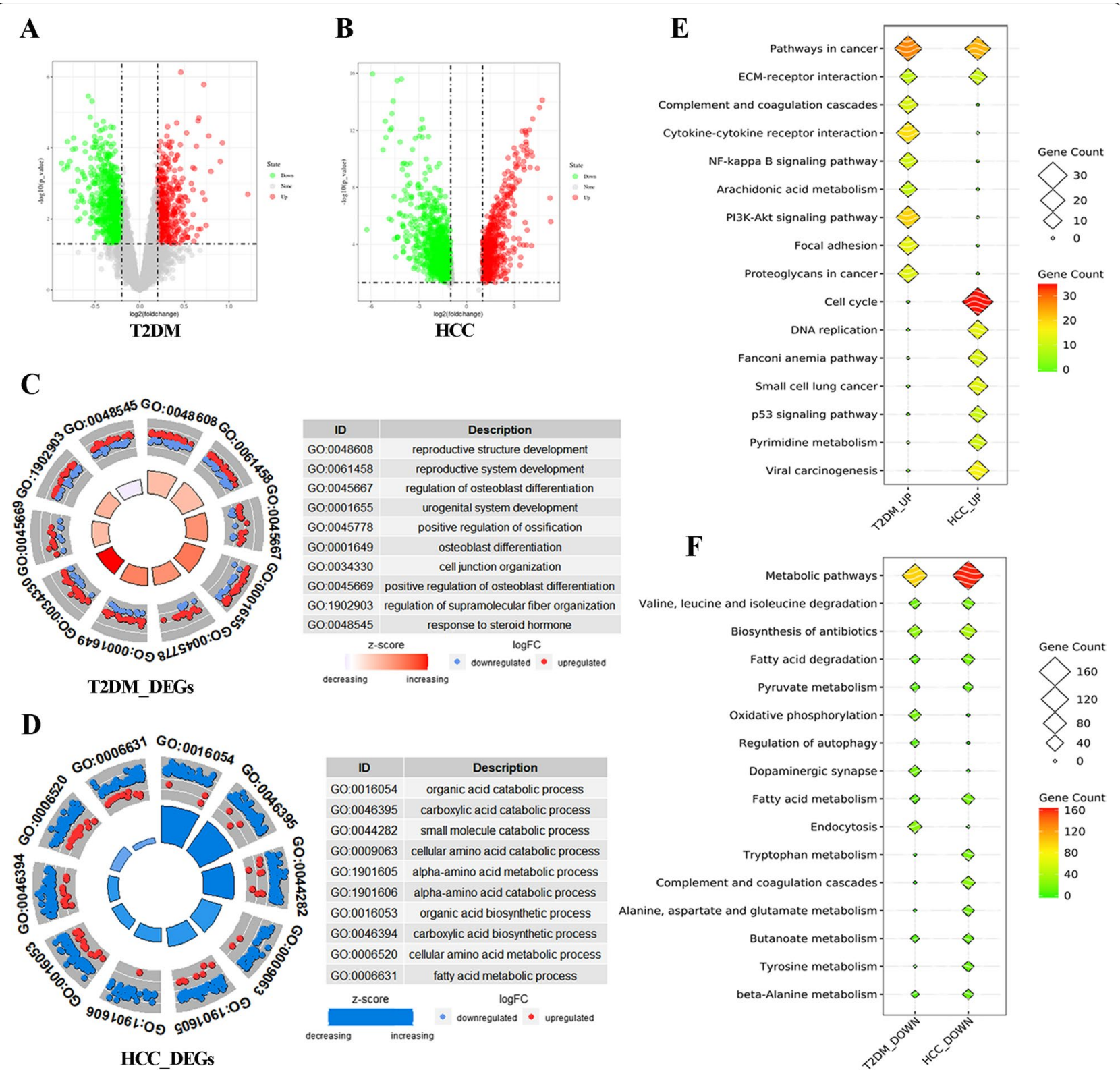

Fig. 1 a and $\mathbf{b}$ The volcano plots of T2DM and HCC. $\mathbf{c}$ and $\mathbf{d}$ The GO terms in T2DM and HCC. $\mathbf{e}$ and $\mathbf{f}$ The KEGG analysis of upregulated genes and downregulated genes in T2DM and HCC

diseases were collected (Supplementary Table S7-S8). These GO terms that summarized the biological functions in each module were analyzed by DOSE package to determine the correlations between T2DM and HCC. As a result, the biological functions of black and pink modules in T2DM focused on cell communication and cell motility, which were similar to those in turquoise module in HCC. Likewise, genes within turquoise and green modules of T2DM were enriched in cell development and cellular metabolism, exhibiting the similar actions to those in blue, brown and yellow modules in HCC (Fig. 2e to g). These modules were considered as significant modules relevant to T2DM and HCC, and were managed with further study. Then, 4 upregulated genes and 33 downregulated genes were obtained through the overlap of several significant modules (Table 1). These 37 genes were annotated via Metascape database, and the result exerted their ability to regulate cellular metabolic processes and liver development (Supplementary Table S9). The associations between genes and diseases were also revealed by DisGeNET (Table 2). The results exhibited that these 
A

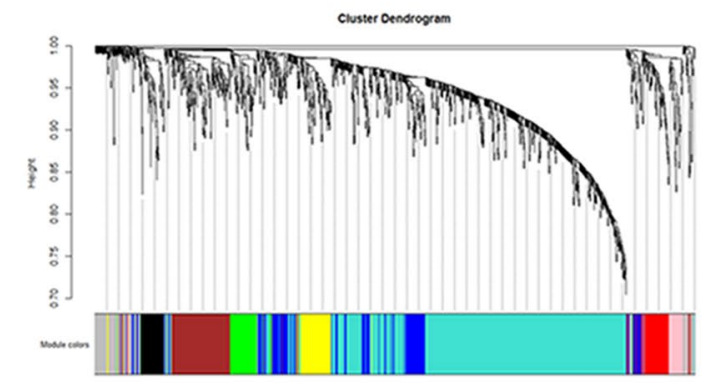

B

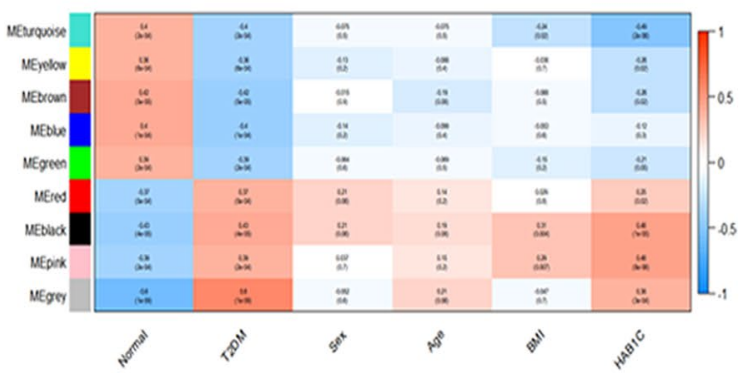

C

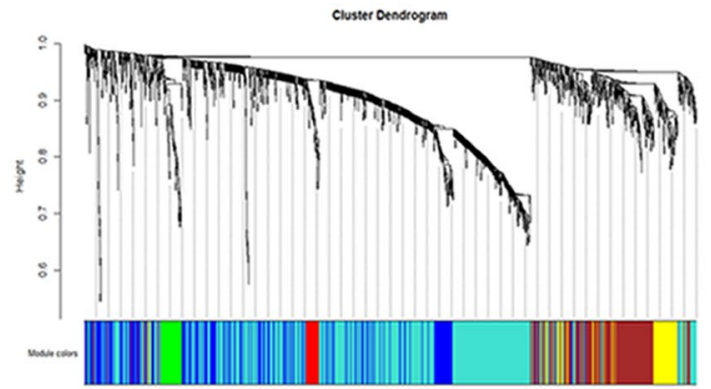

D

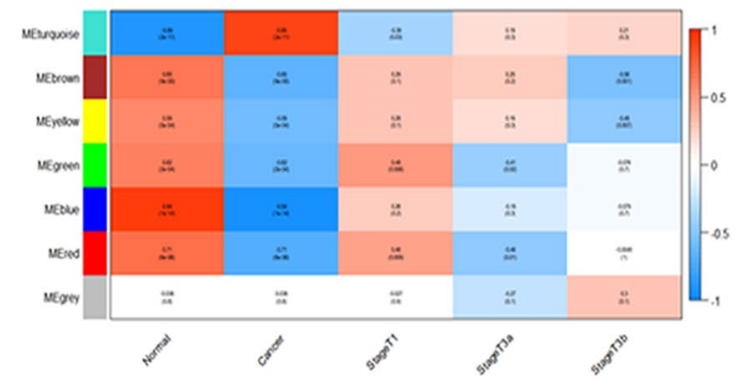

$\mathbf{E}$
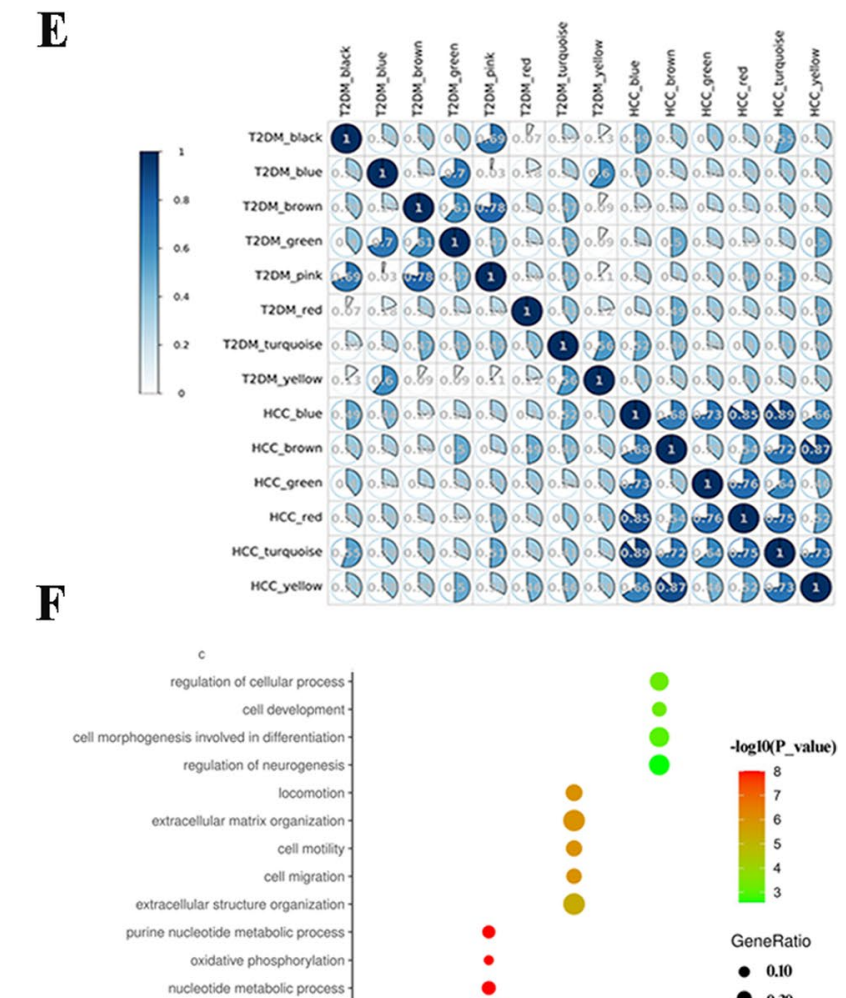

mitocho

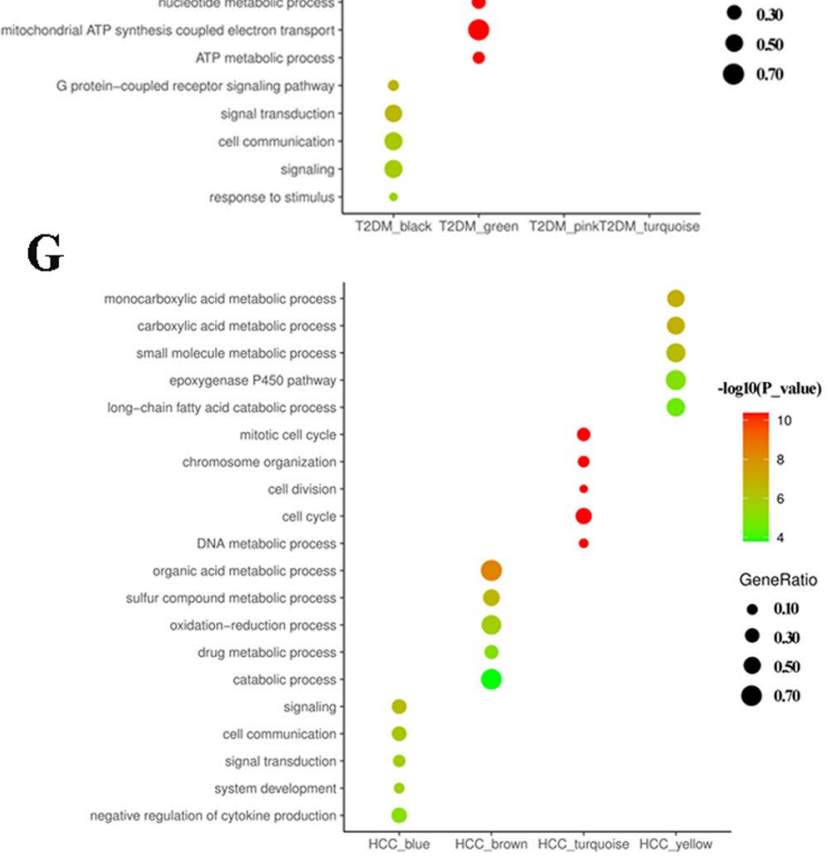

Fig. 2 a and $\mathbf{b}$ Hierarchical clustering dendrogram of T2DM and heatmap plot of correlation between modules and clinical traits of T2DM. c and $\mathbf{d}$ Hierarchical clustering dendrogram of HCC and heatmap plot of correlation between modules and clinical traits of HCC. e The correlation analysis between each module in T2DM and HCC. $\mathbf{f}$ and $\mathbf{g}$ The GO analysis of genes in modules related to T2DM and HCC 
Table 1 Thirty-seven genes correlated with HCC development in T2DM

\begin{tabular}{|c|c|c|c|c|c|}
\hline \multirow[t]{2}{*}{ Gene } & \multirow[t]{2}{*}{ Term } & \multicolumn{2}{|l|}{ T2DM } & \multicolumn{2}{|l|}{$\mathrm{HCC}$} \\
\hline & & LogFC & P_value & LogFC & P_value \\
\hline CXCL5 & C-X-C Motif Chemokine Ligand 5 & 0.40 & 7.57E-03 & 1.65 & 1.95E-02 \\
\hline GPC3 & Glypican 3 & 0.39 & $6.27 \mathrm{E}-03$ & 5.22 & $5.70 \mathrm{E}-08$ \\
\hline NETO2 & Neuropilin And Tolloid Like 2 & 0.22 & $8.83 \mathrm{E}-03$ & 1.39 & $1.14 \mathrm{E}-03$ \\
\hline IGF2BP2 & Insulin Like Growth Factor 2 MRNA Binding Protein 2 & 0.22 & 4.10E-02 & 1.43 & $1.98 \mathrm{E}-02$ \\
\hline ACAT1 & Acetyl-CoA Acetyltransferase 1 & -0.33 & 7.41E-04 & -1.47 & 4.35E-04 \\
\hline CRYL1 & Crystallin Lambda 1 & -0.29 & $1.18 \mathrm{E}-03$ & -1.20 & 4.34E-03 \\
\hline SLC2A2 & Solute Carrier Family 2 Member 2 & -0.65 & $2.54 \mathrm{E}-03$ & -1.88 & 1.13E-02 \\
\hline PCK1 & Phosphoenolpyruvate Carboxykinase 1 & -0.33 & 4.89E-02 & -3.96 & 3.27E-04 \\
\hline ABAT & 4-Aminobutyrate Aminotransferase & -0.50 & $2.75 \mathrm{E}-03$ & -1.91 & 1.15E-02 \\
\hline ACADSB & Acyl-CoA Dehydrogenase Short/Branched Chain & -0.29 & $7.88 \mathrm{E}-04$ & -1.76 & 2.39E-03 \\
\hline ST3GAL6 & ST3 Beta-Galactoside Alpha-2,3-Sialyltransferase 6 & -0.27 & $2.55 \mathrm{E}-02$ & -2.15 & $1.29 \mathrm{E}-06$ \\
\hline EPHX2 & Epoxide Hydrolase 2 & -0.32 & 1.07E-02 & -1.96 & 4.06E-04 \\
\hline KCNMA1 & Potassium Calcium-Activated Channel Subfamily M Alpha 1 & -0.34 & $2.10 \mathrm{E}-02$ & -1.93 & 3.94E-07 \\
\hline SORL1 & Sortilin Related Receptor 1 & -0.42 & $1.01 \mathrm{E}-02$ & -2.36 & 1.36E-07 \\
\hline ACYP2 & Acylphosphatase 2 & -0.25 & $1.51 \mathrm{E}-03$ & -1.09 & 3.55E-03 \\
\hline QDPR & Quinoid Dihydropteridine Reductase & -0.26 & 4.17E-02 & -1.12 & 1.43E-02 \\
\hline TSPAN7 & Tetraspanin 7 & -0.52 & $3.92 \mathrm{E}-03$ & -1.22 & 5.81E-03 \\
\hline MAN1C1 & Mannosidase Alpha Class 1C member 1 & -0.29 & $9.13 \mathrm{E}-03$ & -1.74 & 2.16E-05 \\
\hline SC5D & Sterol-C5-Desaturase & -0.39 & $9.94 \mathrm{E}-04$ & -1.61 & 3.96E-03 \\
\hline IRS2 & Insulin Receptor Substrate 2 & -0.21 & 1.99E-02 & -1.45 & 4.61E-03 \\
\hline RBL2 & RB Transcriptional Corepressor Like 2 & -0.27 & $1.10 \mathrm{E}-02$ & -1.09 & $8.00 \mathrm{E}-03$ \\
\hline F8 & Coagulation factor VIII & -0.20 & $2.28 \mathrm{E}-02$ & -1.23 & 6.47E-04 \\
\hline PRKAR2B & Protein Kinase CAMP-Dependent Type II Regulatory Subunit Beta & -0.30 & $9.63 \mathrm{E}-03$ & -1.56 & $1.25 \mathrm{E}-03$ \\
\hline ETFDH & Electron Transfer Flavoprotein Dehydrogenase & -0.27 & $1.80 \mathrm{E}-02$ & -1.61 & 3.04E-05 \\
\hline LIMCH1 & LIM And Calponin Homology Domains 1 & -0.29 & 4.39E-02 & -1.21 & $1.78 \mathrm{E}-02$ \\
\hline $\mathrm{HADH}$ & 3-Hydroxyacyl-CoA Dehydrogenase & -0.33 & 8.97E-03 & -1.19 & 4.69E-04 \\
\hline ACSL1 & Acyl-CoA Synthetase Long Chain Family Member 1 & -0.43 & $1.36 \mathrm{E}-03$ & -1.63 & 4.18E-03 \\
\hline PTS & 6-Pyruvoyltetrahydropterin Synthase & -0.21 & $4.82 \mathrm{E}-02$ & -1.20 & 1.83E-04 \\
\hline PHLDA2 & Pleckstrin Homology Like Domain Family A Member 2 & 0.29 & $8.18 \mathrm{E}-03$ & 1.47 & 2.22E-02 \\
\hline GAS2 & Growth Arrest Specific 2 & -0.28 & 4.53E-02 & -1.09 & $1.28 \mathrm{E}-02$ \\
\hline TGFBR3 & Transforming Growth Factor Beta Receptor 3 & -0.45 & 2.95E-03 & -1.25 & $1.96 \mathrm{E}-03$ \\
\hline PEMT & Phosphatidylethanolamine N-Methyltransferase & -0.21 & 4.74E-02 & -1.43 & 4.27E-04 \\
\hline PTPN3 & Protein Tyrosine Phosphatase Non-Receptor Type 3 & -0.33 & $1.76 \mathrm{E}-03$ & -1.07 & $2.35 \mathrm{E}-03$ \\
\hline PPP1R1A & Protein Phosphatase 1 Regulatory Inhibitor Subunit 1A & -0.74 & $5.26 \mathrm{E}-05$ & -1.59 & 2.55E-02 \\
\hline RNF130 & Ring Finger Protein 130 & -0.22 & $1.90 \mathrm{E}-03$ & -1.24 & 2.20E-02 \\
\hline RCBTB2 & RCC1 And BTB Domain Containing Protein 2 & -0.30 & $9.65 \mathrm{E}-03$ & -1.26 & 3.23E-05 \\
\hline OAT & Ornithine Aminotransferase & -0.49 & $8.27 \mathrm{E}-04$ & -2.50 & 1.86E-03 \\
\hline
\end{tabular}

37 core genes were highly associated with hypoglycemia, diabetes mellitus, liver carcinoma and steatohepatitis, which were consistent with our study.

\section{Hub genes identification and validation relevant to HCC progression and $\mathrm{HbA} 1 \mathrm{c}$ abnormality}

Notably, genes in the brown and yellow modules negatively associated with StageT3b of HCC and genes in the turquoise cluster negatively correlated with HbA1c of T2DM were also analyzed. A total of 24 genes were identified, of which 12 were selected as hub genes related to tumor invasion and $\mathrm{HbA1c}$ variability. Eight genes, including ACAT1, CRYL1, SLC2A2, PCK1, ABAT, $A C A D S B, S T 3 G A L 6$ and $E P H X 2$ were validated to be negatively correlated with tumor stage and nodal metastasis status based on TCGA database (Fig. 3a to p). Moreover, univariate cox regression analysis was performed to calculate the hazard ratio (HR) between genes and 
Table 2 The relationship between 37 genes and diseases via DisGeNET analysis

\begin{tabular}{llll}
\hline Term & Category & P_value & Enrichment \\
\hline Muscle hypotonia & DisGeNET & $3.98 \mathrm{E}-06$ & 8.3 \\
Hypoglycemia & DisGeNET & $2.51 \mathrm{E}-05$ & 24 \\
Diabetes mellitus, experimental & DisGeNET & $3.16 \mathrm{E}-05$ & 22 \\
Liver carcinoma & DisGeNET & $5.01 \mathrm{E}-05$ & 12 \\
Steatohepatitis & DisGeNET & $5.01 \mathrm{E}-05$ & 41 \\
Seizures & DisGeNET & $6.31 \mathrm{E}-05$ & 6.9 \\
Global developmental delay & DisGeNET & $6.31 \mathrm{E}-05$ & 6.8 \\
Cognitive delay & DisGeNET & $6.31 \mathrm{E}-05$ & 6.8 \\
Mental and motor retardation & DisGeNET & $6.31 \mathrm{E}-05$ & 6.8 \\
Obesity & DisGeNET & $1.00 \mathrm{E}-04$ & 11 \\
\hline
\end{tabular}

survival status of $\mathrm{HCC}$. Genes with $\mathrm{HR}<1$ were defined as protective factors, and the results demonstrated that the increased expressions of five genes, ACAT1, CRYL1, $S L C 2 A 2, P C K 1$ and $A B A T$, might be beneficial to improve the prognosis of $\mathrm{HCC}$ (Fig. 3q).

\section{Prognostic genes confirmation}

The protein expressions of five genes were validated between HCC tissue and normal liver tissue via Human Protein Atlas. The expressions of ACAT1, CRYL1, $S L C 2 A 2, P C K 1$ and $A B A T$ appeared to be lower in HCC issue than in normal tissue (Fig. $4 \mathrm{a}$ and b). Moreover, the expressions of $A C A T 1, S L C 2 A 2, P C K 1$ and $A B A T$ in T2DM samples with HbA1c $>6.5 \%$ were lower than those in normal samples and the samples with HbA1c $\leq 6.5 \%$ (Fig. 4c and d). Besides, PCK1 and ABAT were significantly improved in HepG2 samples prepared with metformin and phenformin in GSE69850, suggesting that glycemic control in T2DM might be beneficial for improving survival outcomes in HCC by increasing the expressions of protective factors (Fig. 4e).

\section{Discussions}

Liver is a major metabolic hub, and aberrant metabolism resulted from T2DM leads to a spectrum of liver dysfunctions such as fatty liver, cirrhosis and even hepatocellular carcinoma. Besides, patients with clinical T2DM characteristics generally influence future HCC and liver-related mortality, particularly those with poorly controlled diabetes [21]. Due to the poor prognosis and unsatisfactory life expectancy in patients with $\mathrm{HCC}$, it is of great clinical significance to clarify the molecular mechanisms of HCC development in T2DM patients.

In this study, key modules and genes involved in T2DM development and HCC growth were observed by bioinformatics method. Through the analysis, it was found that genes were enriched in cell communications and
ECM organization in several significant modules of two diseases; additionally, the staining results showed the increasing deposition of collagen type IV and $\alpha$-smooth muscle actin ( $\alpha$-SMA) in liver of T2DM patients, indicating that hepatic ECM remodeling with overnutrition played a crucial role in liver malignancy [22]. On the other hand, we found that the activity of catabolic progress was decreased in both T2DM and HCC. The pyruvate metabolism and fatty acid metabolism involved in the progress of glycolysis or gluconeogenesis were concurrently enriched in two diseases; not only that, the degradation of amino acid was reduced. These changes may facilitate the biosynthesis and proliferation of cancer cells, which are known as metabolic reprogramming in cancer. Metabolic reprogramming is a hallmark of malignancy, and precedes liver cancer with oncogene mutation [23]. The results of our study suggested that glucose and glutamine metabolism in T2DM was the leading cause of HCC-associated metabolic reprogramming. With further study, 37 genes closely associated with carcinogensis in T2DM were revealed, and the interactions among them were concentrated on carbohydrate metabolism as well. These findings are consistent with several published studies [24, 25], and provide a promising direction for the investigation of the metabolism-related molecules relevant to the development of HCC and progression of T2DM.

Currently, it has been demonstrated that pool glycemic control (assessed by HbA1c) exacerbates HCC. Accumulating evidence indicated that participants with $\mathrm{HbA} 1 \mathrm{c} \geq 6.5 \%$ had an increased risk of cancer mortality and postoperative tumor recurrence $[26,27]$. The signature in this research was based on metabolic genes, which were not only significantly associated with the growth of HCC, but also exerted prediction of the progression and prognosis of $\mathrm{HCC}$ in T2DM patients with higher HbA1c. In present study, we found eight genes including ACAT1, CRYL1, SLC2A2, PCK1, ABAT, $A C A D S B, S T 3 G A L 6$ and $E P H X 2$ were influenced by $\mathrm{HbA1c}$ and related to tumor metastasis. Moreover, the majority of these genes performed on the steps of glutaminolysis, ketogenesis, and molecular transport. Further validation via another database revealed that 4 hub genes, ACAT1, SLC2A2, PCK1 and ABAT, were correlated with tumor survival of $\mathrm{HCC}$ in T2DM with $\mathrm{HbA} 1 \mathrm{c} \geq 6.5 \%$, suggesting the prognostic prediction function of these 4 genes and even new therapeutic targets in HCC.

Among these genes, acetyl-CoA acetyltransferase 1, encoded by $A C A T 1$, is an enzyme that regulates ketone metabolism based on different energy status, and downregulation of $A C A T 1$ is an important feature in the pathophysiology of type 2 diabetes [28]. The decreased ACTA1 


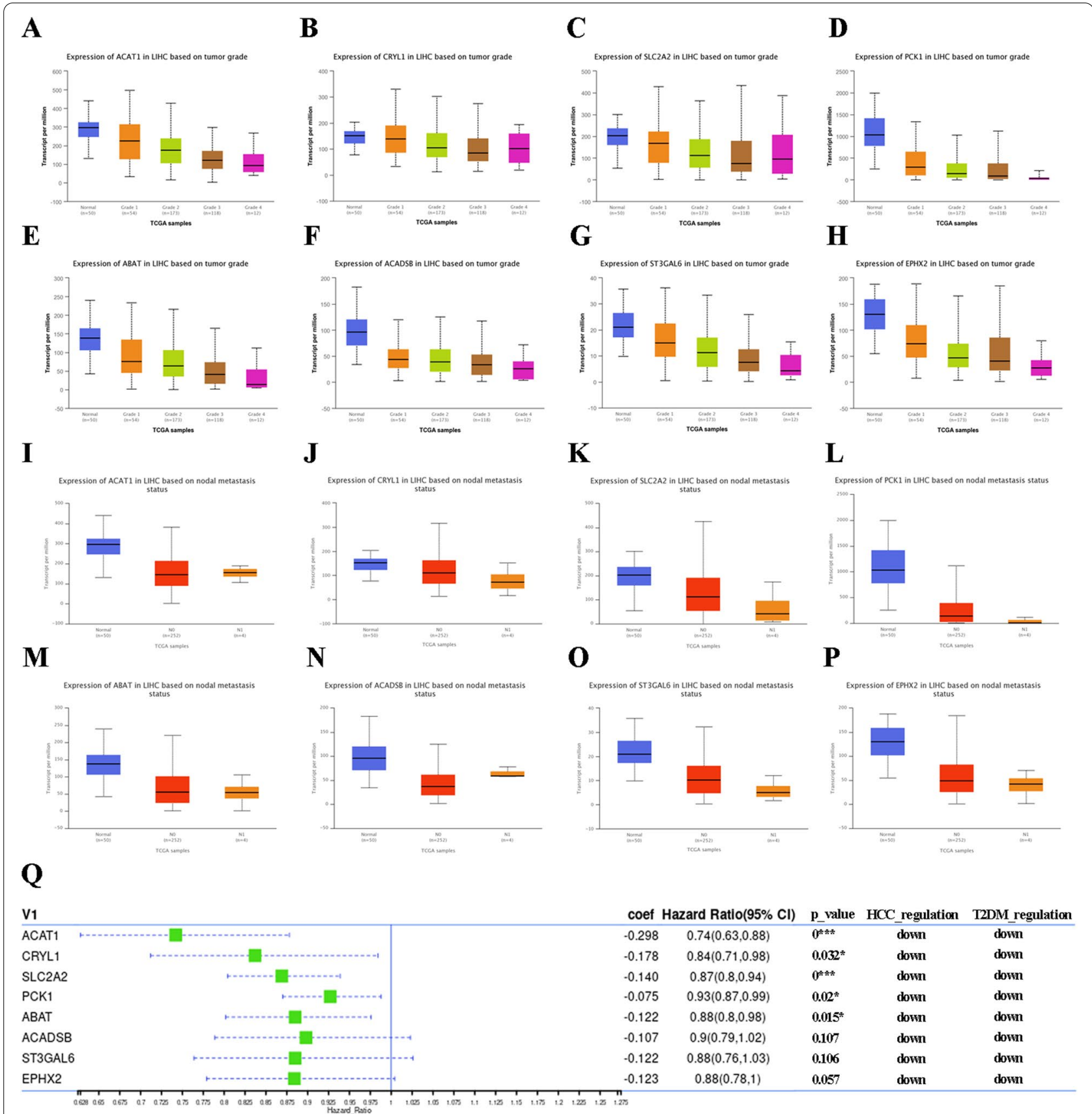

Fig. $\mathbf{3}$ a to $\mathbf{h}$ The expressions of ACAT1, CRYL1, SLC2A2, PCK, ABAT, ACADSB, ST3GAL6 and EPHX2 based on tumor grade. $\mathbf{i}$ to $\mathbf{p}$ The expressions of ACAT1, CRYL1, SLC2A2, PCK1, ABAT, ACADSB, ST3GAL6 and EPHX2 based on metastasis status. q The correlation between genes expression and HCC survival

in late-stage HCC may be caused by metabolic changes, and a number of results showed that over-expression of ACAT1 inhibited the proliferation and migration of tumor cells [29]. However, another study indicated that inhibition of $A T A C 1$ retarded tumor formation in mice combination with sorafenib [30]. The complicated energetics in tumor may be the cause of these contradictory results and it seems that the anti-tumor effect of $A C A T 1$ needs to be further investigated. As a product of $S L C 2 A 2$, glucose transporter 2 is regarded as a glucose sensor due to its low affinity for glucose. The suppression of $S L C 2 A S$ leads to impaired insulin secretion. Studies 
A
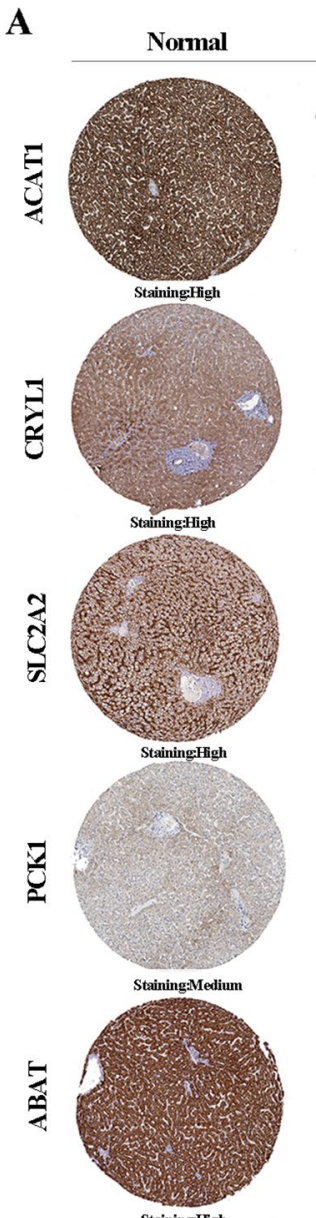

Staining:High
HCC
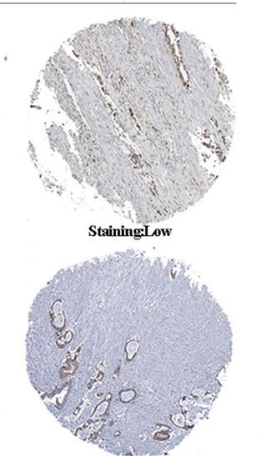

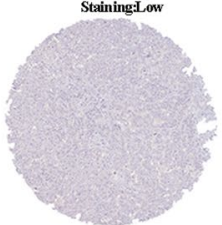
Staining:Not detected

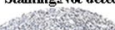

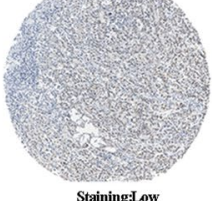
StainingLow

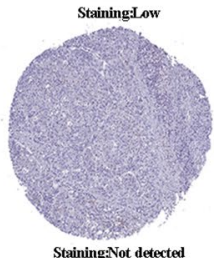

B

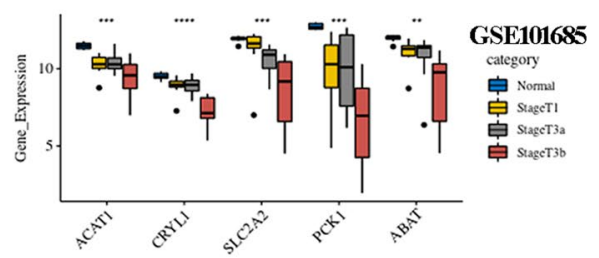

C

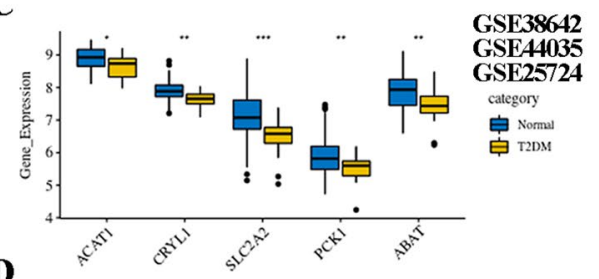

D

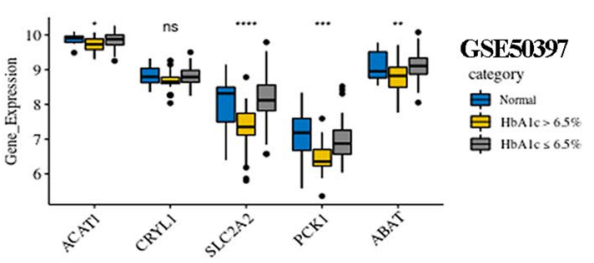

$\mathbf{E}$

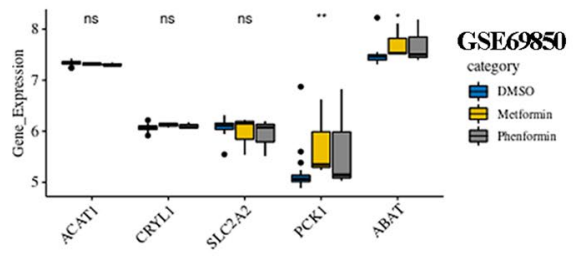

Fig. 4 a The expression level of prognostic genes in HCC and normal samples. $\mathbf{b}$ The expression level of prognostic genes in HCC dataset. c The expression level of prognostic genes in T2DM datasets. $\mathbf{d}$ The expression level of prognostic genes in another T2DM dataset GSE50397. e The expression level of prognostic genes in another HCC dataset GSE69850

have indicated that the expression of $S L C 2 A 2$ is replaced by SLC2A1 in HepG2 cells, resulting in enhanced nutrient uptake and cells proliferation [31]. Phosphoenolpyruvate carboxykinase 1, a post transcriptional protein of PCK1, catalyzes the first rate-limiting reaction of gluconeogenesis in liver, which is closely linked to the tricarboxylic acid cycle (TCA) flux. Numerous studies have proven the anti-tumor effects of $P C K 1$. It has been reported that the viability of HepG2 cells is raised through knocking down $P C K 1$, while increasing the expression of $P C K 1$ activates adenosine $5^{\prime}$-monophosphate-activated protein kinase (AMPK) and c-Jun pathways, blocks gluconeogenesis, and promotes TCA cataplerosis, leading to cell cycle arrest and tumor cells death [32,33]. Moreover, the elevated $P C K 1$ inhibits the migration in two HCC cell lines and the growth of solid tumor in nude mouse xenograft models [34]. ABAT is a gene encoding 4-aminobutyrate aminotransferase. The absence of $A B A T$ in HCC leads to the accumulation of $\gamma$-amino butyric acid (GABA), which promotes the growth of HCC in vitro and in vivo [35]. Recently, $A B A T$ is also essential for mitochondrial nucleoside metabolism, and its dysfunctions enhance cellular nucleoside imbalance and in turn accelerate DNA mutations [36, 37]. Furthermore, mitochondrial nucleoside replication induced by $A B A T$ deficiency also promotes one-carbon metabolism remodeling, which may contribute to cisplatin resistance and cell migration of HCC [38]. Finally, we detected the expressions of prognostic genes in HepG2 cells pre-treated with metformin and phenformin. Interestingly, the expressions of $P C K 1$ and $A B A T$ were increased, suggesting that $P C K 1$ and $A B A T$ were not only the prognostic biomarkers, but also the therapeutic targets of metformin and phenformin in T2DM and HCC. The result was consistent with several studies 
suggesting that metformin remarkably suppressed the growth of PCK1-knockout tumor cells and inhibited tumor growth in an orthotropic HCC mouse model [33].

\section{Conclusions}

In summary, T2DM-associated susceptibility modules and genes for $\mathrm{HCC}$ were revealed through co-expression network analysis. Four key metabolism genes $A C A T 1$, $S L C 2 A 2, P C K 1$ and $A B A T$ were identified from T2DM patients with poorer glycemic control. The dysfunctions of these genes may affect the anabolism and catabolism of substances such as glucose, fatty acids and amino acids, leading to changes in energy sources in cells, and further contributing to the proliferation and migration of HCC. These findings may provide a rational explanation for the higher morbidity and poorer prognosis of HCC in T2DM patients. Additionally, our study has also demonstrated that biguanides may regulate $P C K 1$ and $A B A T$ to achieve therapeutic ends within T2DM and HCC. However, the potential of these genes as effective targets for energy regulation based anti-HCC therapies needs to be verified in more experiments and clinical practice in the future.

\section{Supplementary Information}

The online version contains supplementary material available at https://doi. org/10.1186/s41065-021-00177-x.

Additional file 1:Table S2. The top $100 \mathrm{GO}$ analysis of DEGs in T2DM. Table S2. The top $100 \mathrm{GO}$ analysis of DEGs in HCC. Table S3. The top 20 KEGG analysis of upregulated DEGs inT2DM. Table S4. The top 20 KEGG analysis of downregulated DEGs inT2DM. Table S5. The top 16 KEGG analysis of upregulated DEGs in HCC. Table S6. The top 30 KEGG analysis of downregulated DEGs in HCC. Table S7. The top $10 \mathrm{GO}$ analysis of each module in T2DM. Table S8. The top $10 \mathrm{GO}$ analysis of each module in HCC. Table S9. The top 100 functional analysis of 37 genes via Metascape.

\section{Acknowledgments}

We thank Dr. Jianming Zeng (University of Macau), and all the members of his bioinformatics team, biotrainee, for generously sharing their experience and codes in https://github.com/jmzeng1314.

\section{Authors' contributions}

Yiming Bi and Guanjie Fan conceived this study. Yiming Bi and Bei Yin collected, analyzed and visualized the data. Yiming Bi prepared the manuscript and Guanjie Fan revised the paper. All authors have read and approved the submitted manuscript.

\section{Funding}

This work was supported by the Chinese Government, Ministry of Science and Technology of the People's Republic of China through the National Science and Technology Support Program [Grant number 2015BAI04B09].

\section{Availability of data and materials}

The data of this study are available via corresponding databases mentioned within the article.

\section{Declarations}

Ethics approval and consent to participate Not applicable.
Consent for publication

Not applicable.

\section{Competing interests}

No conflicts of interest in this work are declared by the authors.

\section{Author details}

${ }^{1}$ School of Second Clinical Medicine, Guangzhou University of Chinese Medicine, Guangzhou, China. ${ }^{2}$ Department of Endocrinology, Guangdong Provincial Hospital of Chinese Medicine, the Second Affiliated Hospital of Guangzhou University of Chinese Medicine, Guangzhou, China.

Received: 8 October 2020 Accepted: 4 April 2021

Published online: 17 April 2021

\section{References}

1. Saeedi P, Petersohn I, Salpea P, Malanda B, Karuranga S, Unwin N, et al. Global and regional diabetes prevalence estimates for 2019 and projections for 2030and 2045: results from the International Diabetes Federation Diabetes Atlas, 9thedition. Diab Res Clin Pract. 2019;157:107843.

2. Calle EE, Rodriguez C, Walker-Thurmond K, Thun MJ. Overweight, obesity, and mortality from cancer in a prospectively studied cohort ofU.S. adults. N Engl J Med. 2003;348(17):1625-38.

3. Baecker A, Liu X, La Vecchia C, Zhang ZF. Worldwide incidence of hepatocellular carcinoma cases attributable to major risk factors. Eur J Cancer Prev. 2018;27(3):205-12.

4. El-Serag HB, Tran T, Everhart JE. Diabetes increases the risk of chronic liver disease and hepatocellular carcinoma. Gastroenterology. 2004;126(2):460-8.

5. Simon TG, King LY, Chong DQ, Nguyen LH, Ma Y, VoPham T, et al. Diabetes, metabolic comorbidities, and risk of hepatocellular carcinoma: results from two prospective cohort studies. Hepatology. 2018;67(5):1797-806.

6. Mantovani A, Targher G. Type 2 diabetes mellitus and risk of hepatocelIular carcinoma: spotlight on nonalcoholic fatty liver disease. Ann Transl Med. 2017;5(13):270.

7. Harding JL, Shaw JE, Peeters A, Cartensen B, Magliano DJ. Cancer risk among people with type 1 and type 2 diabetes: disentangling true associations, detection bias, and reverse causation. Diabetes Care. 2015;38(2):264-70.

8. Schulte L, Scheiner B, Voigtländer T, Koch S, Schweitzer N, Marhenke S, et al. Treatment with metformin is associated with a prolonged survival in patients with hepatocellular carcinoma. Liver Int. 2019;39(4):714-26.

9. Bonagiri PR, Shubrook JH. Review of associations between type 2 diabetes and cancer. Clin Diabetes. 2020;38(3):256-65.

10. Shlomai G, Neel B, LeRoith D, Gallagher EJ. Type 2 diabetes mellitus and cancer: The role of pharmacotherapy. J Clin Oncol. 2016;34(35):4261-9.

11. Singh MK, Das BK, Choudhary S, Gupta D, Patil UK. Diabetes and hepatocellular carcinoma: a pathophysiological link and pharmacological management. Biomed Pharmacother. 2018;106:991-1002.

12. Hu CM, Tien SC, Hsieh PK, Jeng YM, Chang MC, Chang YT, et al. High Glucose triggers nucleotide imbalance through O-GlcNAcylation of key enzymes and induces KRAS mutation in pancreatic cells. Cell Metab. 2019;29(6):1334-49.

13. Langfelder P, Horvath S. WGCNA: an R package for weighted correlation network analysis. BMC Bioinformatics. 2008:9:559.

14. Zhu Y, Ding X, She Z, Bai X, Nie Z, Wang F, et al. Exploring shared pathogenesis of Alzheimer's disease and type 2 diabetes mellitus via coexpression networks analysis. Curr Alzheimer Res. 2020;17(6):566-75.

15. Bi N, Sun Y, Lei S, Zeng Z, Zhang Y, Sun C, et al. Identification of 40 S ribosomal protein $\mathrm{S} 8$ as a novel biomarker for alcohol-associated hepatocellular carcinoma using weighted gene co-expression network analysis. Oncol Rep. 2020;44(2):611-27.

16. Ritchie ME, Phipson B, Wu D, Hu Y, Law CW, Shi W, et al. limma powers differential expression analyses for RNA-sequencing and microarray studies. Nucleic Acids Res. 2015;43(7):e47.

17. Ye Z, Zeng Z, Wang D, Lei S, Shen Y, Chen Z. Identification of key genes associated with the progression of intrahepatic cholangiocarcinoma 
using weighted gene co-expression network analysis. Oncol Lett. 2020;20(1):483-94.

18. Yu G, Li F, Qin Y, Bo X, Wu Y, Wang S. GOSemSim: an R package for measuring semantic similarity among $\mathrm{GO}$ terms and gene products. Bioinformatics. 2010;26(7):976-8.

19. Zhou Y, Zhou B, Pache L, Chang M, Khodabakhshi AH, Tanaseichuk O, et al. Metascape provides a biologist-oriented resource for the analysis of systems-level datasets. Nat Commun. 2019;10(1):1523.

20. Chandrashekar DS, Bashel B, Balasubramanya S, Creighton CJ, PonceRodriguez I, Chakravarthi BVSK, et al. UALCAN: a portal for facilitating tumor subgroup gene expression and survival analyses. Neoplasia. 2017;19(8):649-58.

21. Hsiang JC, Gane EJ, Bai WW, Gerred SJ. Type 2 diabetes: a risk factor for liver mortality and complications in hepatitis B cirrhosis patients. J Gastroenterol Hepatol. 2015;30(3):591-9.

22. Jaskiewicz K, Rzepko R, Sledzinski Z. Fibrogenesis in fatty liver associated with obesity and diabetes mellitus type 2. Dig Dis Sci. 2008;53(3):785-8.

23. Hu S, Balakrishnan A, Bok RA, Anderton B, Larson PE, Nelson SJ, et al. 13C-pyruvate imaging reveals alterations in glycolysis that precede c-Myc-induced tumor formation and regression. Cell Metab. 2011;14(1):131-42.

24. Berndt N, Egners A, Mastrobuoni G, Vvedenskaya O, Fragoulis A, Dugourd $A$, et al. Kinetic modelling of quantitative proteome data predicts metabolic reprogramming of liver cancer. Br J Cancer. 2020;122(2):233-44.

25. Dai W, Xu L, Yu X, Zhang G, Guo H, Liu H, et al. OGDHL silencing promotes hepatocellular carcinoma by reprogramming glutamine metabolism. J Hepatol. 2020;72(5):909-23.

26. Bancks MP, Odegaard AO, Pankow JS, Koh WP, Yuan JM, Gross MD, et al. Glycated hemoglobin and all-cause and cause-specific mortality in Singaporean Chinese without diagnosed diabetes: the Singapore Chinese Health Study. Diab Care. 2014;37(12):3180-7.

27. Kaneda K, Uenishi T, Takemura S, Shinkawa H, Urata Y, Sakae M, et al. The influence of postoperative glycemic control on recurrence after curative resection in diabetics with hepatitis $C$ virus-related hepatocellular carcinoma. J Surg Oncol. 2012;105(6):606-11.

28. Dharuri H, T Hoen PA, van Klinken JB, Henneman P, Laros JF, Lips MA, et al. Downregulation of the acetyl-CoA metabolic network in adipose tissue of obesediabeticindividuals and recovery after weight loss. Diabetologia. 2014;57(11):2384-92.

29. Chen L, Peng T, Luo Y, Zhou F, Wang G, Qian K, et al. ACAT1 and metabolism-related pathways are essential for the progression of clear cell renal cell carcinoma (ccRCC), as determined by co-expression network analysis. Front Oncol. 2019;9:957.

30. Gu L, Zhu Y, Lin X, Tan X, Lu B, Li Y. Stabilization of FASN by ACAT1-mediated GNPAT acetylation promotes lipid metabolism and hepatocarcinogenesis. Oncogene. 2020;39(11):2437-49.

31. Lei Y, Hu Q, Gu J. Expressions of carbohydrate response element binding protein and glucose transporters in liver cancer and clinical significance. Pathol Oncol Res. 2020;26(2):1331-40.

32. Liu MX, Jin L, Sun SJ, Liu P, Feng X, Cheng ZL, et al. Metabolic reprogramming by PCK1 promotes TCA cataplerosis, oxidative stress and apoptosis in liver cancer cells and suppresses hepatocellular carcinoma. Oncogene. 2018;37(12):1637-53.

33. Tuo L, Xiang J, Pan $X$, Hu J, Tang H, Liang L, et al. PCK1 negatively regulates cell cycle progression and hepatoma cell proliferation via the AMPK p27(Kip1) axis. J Exp Clin Cancer Res. 2019;38(1):50.

34. Tang Y, Zhang Y, Wang C, Sun Z, Li L, Cheng S, et al. Overexpression of PCK1 gene antagonizes hepatocellular carcinoma through the activation of gluconeogenesis and suppression of glycolysis pathways. Cell Physiol Biochem. 2018:47(1):344-55

35. Li YH, Liu Y, Li YD, Liu YH, Li F, Ju Q, et al. GABA stimulates human hepatocellular carcinoma growth through overexpressed GABAA receptor theta subunit. World J Gastroenterol. 2012;18(21):2704-11.

36. Besse A, Wu P, Bruni F, Donti T, Graham BH, Craigen WJ, et al. The GABA transaminase, $A B A T$, is essential for mitochondrial nucleoside metabolism. Cell Metab. 2015;21(3):417-27.

37. Hsu CC, Lee HC, Wei YH. Mitochondrial DNA alterations and mitochondrial dysfunction in the progression of hepatocellular carcinoma. World J Gastroenterol. 2013;19(47):8880-6.
38. Nikkanen J, Forsström S, Euro L, Paetau I, Kohnz RA, Wang L, et al. Mitochondrial DNA replication defects disturb cellular dNTP pools and remodel one-carbon metabolism. Cell Metab. 2016;23(4):635-48.

\section{Publisher's Note}

Springer Nature remains neutral with regard to jurisdictional claims in published maps and institutional affiliations.
Ready to submit your research? Choose BMC and benefit from:

- fast, convenient online submission

- thorough peer review by experienced researchers in your field

- rapid publication on acceptance

- support for research data, including large and complex data types

- gold Open Access which fosters wider collaboration and increased citations

- maximum visibility for your research: over 100M website views per year

At BMC, research is always in progress.

Learn more biomedcentral.com/submissions 